ORIGINAL ARTICLE

\title{
Clinical validity of plasma and urinary desmosine as biomarkers for chronic obstructive pulmonary disease
}

\author{
Jeffrey T-J Huang, ${ }^{1,2,3}$ Rekha Chaudhuri, ${ }^{4}$ Osama Albarbarawi, ${ }^{1}$ Alun Barton, ${ }^{1}$ \\ Christal Grierson, ${ }^{1}$ Petra Rauchhaus, ${ }^{1}$ Christopher J Weir, ${ }^{5}$ Martina Messow, \\ Nicola Stevens ${ }^{3}{ }^{3}$ Charles McSharry, ${ }^{4}$ Giora Feuerstein, ${ }^{2}$ Somnath Mukhopadhyay, ${ }^{7}$ \\ Jeffrey Brady, ${ }^{1,2,3}$ Colin N A Palmer, ${ }^{3}$ Douglas Miller, ${ }^{2}$ Neil C Thomson ${ }^{4}$
}

- Additional materials are published online only. To view these files please visit the journal online (http://thorax.bmj. com/content/67/6.toc)

${ }^{1}$ Translational Medicine Research Collaboration, Dundee,

${ }^{2}$ Clinical Research, Pfizer Worldwide Research \& Development, Collegeville, Pennsylvania, USA

${ }^{3}$ Medical Research Institute School of Medicine, University of Dundee, Dundee, UK

${ }^{4}$ Institute of Infection, Immunity \& Inflammation, University of Glasgow, Glasgow, UK ${ }^{5}$ MRC Hub for Trials Methodology Research, University of Edinburgh, Edinburgh, UK

${ }^{6}$ Robertson Centre for Biostatistics, University of Glasgow, Glasgow, UK ${ }^{7}$ Academic Department of Paediatrics, Brighton and Sussex Medical School, Brighton, UK

\section{Correspondence to}

Dr Jeffrey T-J Huang, Medical Research Institute, School of Medicine, University of Dundee, Dundee, DD1 9SY, UK: j.t.j.huang@dundee.ac.uk

Received 1 April 2011 Accepted 9 December 2011 Published Online First 16 January 2012

\begin{abstract}
Background Although an increased concentration of degraded elastin products in patients with chronic obstructive pulmonary disease (COPD) has been reported for many years, its clinical validity and utility remain uncertain due to technical difficulties, small study groups and the unknown relationship between exacerbation and elastin degradation. The objectives of this study were to determine the validity of urinary and blood total desmosine/isodesmosine in patients with COPD and asthma and to evaluate their relationship to exacerbation status and lung function.
\end{abstract}

Methods Urinary and blood desmosine levels were measured using validated isotopic dilution liquid chromatography-tandem mass spectrometry methods. Results 390 study participants were recruited from the following groups: healthy volunteers, stable asthma, stable and 'during an exacerbation' COPD. Compared with healthy non-smokers, we found increased urinary or blood desmosine levels in patients with COPD, but no differences in patients with asthma or healthy smokers. The elevation of urinary desmosine levels was associated with the exacerbation status in patients with COPD. Approximately $40 \%$ of patients with stable and 'during an exacerbation' COPD showed elevated blood desmosine levels. Blood desmosine levels were strongly associated with age and were negatively correlated with lung diffusing capacity for carbon monoxide.

Conclusion The results suggest that urinary desmosine levels are raised by exacerbations of COPD whereas blood desmosine levels are elevated in a subgroup of patients with stable COPD and reduced lung diffusing capacity. The authors speculate that a raised blood desmosine level may identify patients with increased elastin degradation suitable for targeted therapy. Future prospective studies are required to investigate this hypothesis.

\section{INTRODUCTION}

Pulmonary emphysema, the destruction of elastic tissue in alveolar septae of the lung, is known to affect $12-28 \%$ of patients with chronic obstructive pulmonary disease (COPD) ${ }^{1}$ and is thought to represent an end result of the proteinaseantiproteinase imbalance associated with aberrant inflammation. ${ }^{2}$ There is no early diagnostic tool available to stratify at-risk patients with elevated elastin degradation. Such a tool could be critical for

\section{Key messages}

What is the key question?

- Are urinary and plasma desmosine useful biomarkers of disease in chronic obstructive pulmonary disease (COPD) and asthma, taking account of smoking and exacerbation status.

What is the bottom line?

- Plasma desmosine concentrations are elevated in patients with stable COPD but reduced lung diffusing capacity. Urinary desmosine concentrations are raised during exacerbations of COPD.

\section{Why read on?}

- This study using improved validity of analytical assays and substantial patient numbers describes the value of urinary and plasma desmosine as a biomarker of disease activity in COPD.

the development of intervention strategies to prevent disease progression.

The analysis of turnover of mature elastin may offer promising potential as a surrogate marker for lung destruction and for testing the efficacy of agents with the potential to reduce elastin breakdown. Two isomeric pyridinium-based amino acids, desmosine (DES) and isodesmosine (IDS) resulting from the condensation of four lysine residues between elastin proteins by lysyl-oxidase, represent ideal biomarkers for monitoring elastin turnover because these special cross-links are only found in mature elastin in mammals. ${ }^{3}$ The release of these two compounds and DES-containing or IDScontaining peptides into body fluids has been used to estimate levels of elastin degradation in diseases such as COPD. ${ }^{4}$ Despite being one of the most promising biomarkers linking directly to the pathophysiology, the clinical validity and utility of urine and blood total DES/IDS (uDES and bDES) as biomarkers for COPD remains unproven. The major reasons are issues related to analytical validity (including sensitivity, specificity, inaccuracy) of assays, small study groups, ${ }^{4}$ and the lack of large longitudinal studies to determine the predictive power of uDES and bDES for patients' clinical outcome. online under the BMJ Journals unlocked scheme, see http:// thorax.bmi.com/site/about/ unlocked.xhtml 
Analytical specificity has been one of the key problems for methodologies such as immunoassays, ${ }^{5-8}$ capillary electrophoresis, $^{9-12}$ electrokinetic chromatography, ${ }^{13}$ and to a lesser degree, high-performance liquid chromatography isotope dilution. ${ }^{14-17}$ Liquid chromatography-tandem mass spectrometry (LC-MS/MS) methods are believed to provide the best specificity and sensitivity. ${ }^{4}$ However, mass spectrometry is not intrinsically quantitative-often matrix compounds in the complex samples interact with the analytes in the MS ion source resulting in variability that adversely impacts data quality. To address this issue, our laboratory has developed and analytically validated reliable isotope dilution LC-MS/MS methods for DES/IDS measurement. ${ }^{18}$

Using these validated methods, the primary aim of this study was to examine the clinical validity of $\mathrm{UDES}$ and bDES as phenotyping biomarkers for COPD. The secondary goals were to investigate the relationships of $\mathrm{uDES}$ and bDES levels to smoking status, disease entity, severity, exacerbations and lung function.

\section{METHODS}

\section{Study participants}

Volunteers were recruited from the hospital respiratory clinics and from general practices in Glasgow (Glasgow COPD and Asthma Biomarker study; group 1) and Dundee (group 2). Potentially eligible patients were identified by scrutiny of hospital records, from general practice asthma clinic registers, by advertisements in local papers and from computerised general practice records. For group 1, 306 patients were screened for the study and 204 met the required inclusion and exclusion criteria. All study participants gave their written informed consent and the original study was approved by the local ethics committees and Institutional Review Board.

Participants were recruited from the following groups:

- Stable COPD: age range 40-80 years and duration of COPD $\geq 6$ months; symptoms of cough and breathlessness, history of smoking over 10 pack years; forced expiratory volume in $1 \mathrm{~s}\left(\mathrm{FEV}_{1}\right) /$ forced vital capacity (FVC) post bronchodilator $<70 \%$; $\mathrm{FEV}_{1} 30-80 \%$ predicted with reversibility to $2.5 \mathrm{mg}$ salbutamol of $<200 \mathrm{ml}$; exacerbation free for 4 weeks and no chest infection for 2 weeks.

- COPD during an exacerbation: age range 40-80 years and duration of COPD $\geq 6$ months; symptoms of cough and breathlessness, current or past history of smoking $>10$ pack years; all participants were hospitalised because of the exacerbation.

- Stable asthma: age range 18-75 years and duration of asthma $\geq 6$ months; symptoms of episodic wheezing chest tightness and/or dyspnoea (see online supplementary information for further details); on stable medication for 4 weeks and no exacerbation for 4 weeks or chest infection for 2 weeks; definition of smokers, $\geq 10$ pack years and currently smoking at least give cigarettes per day.

- Healthy controls (smokers and non-smokers): age range 18-80 years; no known respiratory disease or recent (in last 2 weeks) chest infection; normal spirometry (FEV $1 / F V C$ $>80 \% ; \mathrm{FEV}_{1}>80 \%$ predicted); no chronic respiratory symptoms; no known exposure to passive smoke in the $4 \mathrm{~h}$ prior to performing the tests in healthy non-smokers based on history and further examination by serum cotinine level (median (IOR: Q1-O3) $=0.9(0.3-1.1) \mathrm{ng} / \mathrm{ml})$.

\section{Sample collection}

The majority of participants (group 1) had spot urine, blood and sputum samples collected during two visits to establish the stability of uDES and bDES levels in each group. The remaining participants (group 2) had spot urine and sputum or blood samples collected (see online supplementary information for further details).

\section{uDES, bDES and sputum matrix metalloproteinase-9 activity assays}

uDES and urinary creatinine assays were carried out using validated isotope dilution LC-MS/MS methods. ${ }^{18}$ Similarly bDES concentration was analysed using a modified assay with 10 times the lower limit of quantification. The uDES levels were expressed as uDES concentration normalised to urinary creatinine concentration according to previous reports. ${ }^{4}$ The matrix metalloproteinase-9 (MMP-9) activity kit was purchased from $\mathrm{R} \& \mathrm{D}$ systems (Abingdon, UK) and has been validated for use in sputum supernatant. The assay performance can be found in supplementary online table E1.

\section{Lung function measurements}

Spirometry was performed to American Thoracic Society guidelines. Transfer factor of the lung for carbon monoxide (TLCO\%) was measured using the body box technique (Zan500 Body Plethysmography, nSpire Health Ltd, Hertford, UK) and corrected for haemoglobin and carboxy haemoglobin in patients with stable asthma and COPD.

\section{Data analysis}

Peak area under the curve for DES/IDS and $\mathrm{D}_{5}-\mathrm{DES}$, calibration curve, $\mathrm{SD}$, coefficient of variation $(\mathrm{CV})$ and \% bias were generated and calculated by LCquan (ThermoFisher, Hemel Hempstead, UK). Data normality was examined using the Shapiro-Wilk test to determine the use of parametric or non-parametric tests. Two sample t test, Mann-Whitney $U$ test, one-way analysis of variance (ANOVA), Kruskal-Wallis ANOVA and Spearman correlation were performed using Prism 5.04 or SPSS V.17.0 as appropriate.

\section{RESULTS}

\section{Clinical characteristics of study groups}

We investigated uDES and bDES levels in a total of 390 participants in two study groups: group 1 comprised five subgroups, including 26 healthy non-smokers, 20 healthy smokers, 53 stable asthma non-smokers, 56 stable asthma smokers, and 53 patients with stable COPD (table 1); group 2 comprised two subgroups including 81 healthy volunteers and 105 patients with COPD at the time of an exacerbation (table 2). In the asthma and stable COPD groups, patients with different severities (ie, mild, moderate and severe) were equally distributed so that the relationship between DES levels and increasing disease severity can be investigated. The average age of patients with COPD in group 1 and group 2 (urine and sputum) was significantly older compared with the other groups $(p<0.001$, table 1 and 2$)$. There was a higher percentage of men in the healthy volunteers in the blood samples of group 2 .

\section{uDES levels in patients with stable asthma, stable and 'during an exacerbation' COPD and healthy volunteers}

In group 1, there was no significant difference in $\mathrm{uDES}$ levels between smokers and non-smokers in both healthy volunteers and patients with asthma (figure $1 \mathrm{~A}$ and table 1). In addition, we did not observe a difference between healthy volunteers and patients with asthma (figure $1 \mathrm{~A}$ and table 1). uDES levels were not associated with serum cotinine levels in healthy volunteers $(\mathrm{r}=0.02$, Spearman correlation). 
Table 1 Demographic and desmosine data for group 1 consisting of healthy volunteers and patients with stable chronic obstructive pulmonary disease (COPD) and asthma

\begin{tabular}{|c|c|c|c|c|c|}
\hline \multirow{3}{*}{$\begin{array}{l}\text { Sample type } \\
\text { Group }\end{array}$} & \multicolumn{5}{|l|}{ Group 1} \\
\hline & \multicolumn{5}{|c|}{ Blood, urine and sputum } \\
\hline & $\begin{array}{l}\text { Healthy volunteers: } \\
\text { non-smokers }\end{array}$ & $\begin{array}{l}\text { Healthy volunteers: } \\
\text { smokers }\end{array}$ & $\begin{array}{l}\text { Asthma: } \\
\text { non-smokers }\end{array}$ & $\begin{array}{l}\text { Asthma: } \\
\text { smokers }\end{array}$ & Stable COPD \\
\hline Number of participants & 26 & 20 & 53 & 56 & 53 \\
\hline Gender (M/F) & $8 / 18$ & $9 / 11$ & $22 / 31$ & $27 / 29$ & $23 / 30$ \\
\hline $\begin{array}{l}\text { Smoking status (smokers/ } \\
\text { ex-smokers/non-smokers) }\end{array}$ & $0 / 0 / 26$ & $20 / 0 / 0$ & $0 / 0 / 53$ & $56 / 0 / 0$ & $28 / 25 / 0$ \\
\hline Age (years) & $51 \pm 13$ & $52 \pm 9$ & $46 \pm 12$ & $47 \pm 10$ & $65 \pm 7^{*}$ \\
\hline Body mass index & $27(22-28)$ & $27(21-30)$ & $28(25-36)$ & $26(23-30)$ & $27(23-30)$ \\
\hline $\mathrm{FEV}_{1}(\%$ predicted $)$ & $106(95-115)$ & $93(88-100)$ & $79(70-91) \dagger$ & $78(58-93) \dagger$ & $62(44-77) \ddagger$ \\
\hline $\mathrm{TLCO} \% \mathrm{COHb}$ & NA & NA & $86 \pm 11$ & $77 \pm 18 \S$ & $60 \pm 15 \Phi$ \\
\hline uDES (ng/mg creatinine) & $9(8-13)$ & $11(9-15)$ & $8(6-10)$ & $10(7-13)$ & $11(9-15)^{* *}$ \\
\hline bDES (ng/ml) & $0.21(0.19-0.24)$ & $0.23(0.19-0.26)$ & $0.21(0.19-0.25)$ & $0.22(0.19-0.25)$ & $0.29(0.24-0.35)^{*}$ \\
\hline
\end{tabular}

Data are shown as median (IOR) or mean \pm SD based on whether data were normally distributed. Kruskal-Wallis analysis of variance (ANOVA) with Dunn's method for pairwise multiple comparison or one-way ANOVA with Tukey's method was used.

${ }^{*} p<0.001$, versus other four groups.

$t p<0.05$, versus healthy non-smokers and smokers.

$\neq p<0.001$ versus healthy non-smokers and smokers; $p<0.05$ versus non-smokers and smokers with asthma.

$\S \mathrm{p}<0.001$, versus non-smokers with asthma and patients with COPD.

$\lceil\mathrm{p}<0.001$, versus non-smokers and smokers with asthma.

${ }^{* *} \mathrm{p}<0.01$ versus non-smokers with asthma.

bDES, blood desmosine; $\mathrm{FEV}_{1}$, forced expiratory volume in $1 \mathrm{~s}$; TLCO\%COHb, transfer factor corrected for haemoglobin and carboxy haemoglobin; uDES, urinary desmosine.

In COPD groups, patients with stable COPD excreted a $\sim 37 \%$ higher level of uDES compared with non-smokers with asthma ( $p<0.01$, group 1 ) but no difference was found between the stable COPD group and healthy non-smokers or smokers (table 1). Similar results were found at a subsequent visit (see online supplementary table E2). Patients with 'during an exacerbation' COPD, however, had significantly higher uDES levels compared with other subgroups $(p<0.001$, figure $1 \mathrm{~A}$ and table 2). No significant difference was found between nonsmokers, current smokers and ex-smokers in the 'during an exacerbation' COPD group ( $\mathrm{p}=0.11$; see online supplementary table E3). There was no correlation between uDES levels and age, gender or body mass index (data not shown). These results suggest that the elevation of uDES levels only occurs in COPD at the time of an exacerbation, whereas during clinical stable disease, the uDES levels remain normal.

\section{bDES levels in patients with stable asthma, stable and exacerbated COPD and healthy volunteers}

Similar to the findings on uDES, the bDES levels were no different among healthy smokers and non-smokers, smokers with asthma and non-smokers with asthma in group 1 (table 1 and figure 1B). bDES levels were not significantly correlated with serum cotinine levels in healthy volunteers $(r=0.01$, Spearman correlation). Interestingly, in contrast to little or no change in uDES levels, the bDES levels in these patients with stable COPD were significantly elevated compared with healthy non-smokers and smokers $(p<0.001$, table 2 and figure $1 \mathrm{~B})$. The bDES levels between patients with stable and 'during an exacerbation' COPD were similar. Despite the distinct distribution of these two measurements according to the patient's exacerbation status, the levels of $\mathrm{UDES}$ and bDES were found to be positively correlated in both stable $(\mathrm{r}=0.33, \mathrm{p}<0.001$, Spearman

Table 2 Demographic and desmosine data for group 2 consisting of healthy volunteers and patients with an exacerbation of chronic obstructive pulmonary disease (COPD)

\begin{tabular}{|c|c|c|c|c|}
\hline \multirow{3}{*}{$\begin{array}{l}\text { Sample type } \\
\text { Group }\end{array}$} & \multicolumn{4}{|l|}{ Group 2} \\
\hline & \multicolumn{2}{|l|}{ Urine and sputum } & \multicolumn{2}{|l|}{ Blood } \\
\hline & Healthy volunteers (HV2a) & $\begin{array}{l}\text { Patients with 'during } \\
\text { an exacerbation' COPD }\end{array}$ & Healthy volunteers (HV2b) & $\begin{array}{l}\text { Patients with 'during } \\
\text { an exacerbation' COPD }\end{array}$ \\
\hline Number of participants & 62 & $50^{*}$ & 19 & $102^{*}$ \\
\hline Gender (M/F) & $24 / 38$ & $24 / 26$ & $18 / 1$ & $43 / 59$ \\
\hline $\begin{array}{l}\text { Smoking status (smokers/e-smokers/ } \\
\text { non-smokers/unknown) }\end{array}$ & $13 / 41 / 8 / 0$ & $31 / 2 / 15 / 2$ & $10 / 0 / 9 / 0$ & $55 / 33 / 0 / 14$ \\
\hline $\mathrm{FEV}_{1}(\%$ predicted) & $103 \pm 13$ & $39 \pm 16 \dagger$ & NA & $47 \pm 18$ \\
\hline uDES (ng/mg creatinine) & $8(6-10)$ & $16(14-22) \dagger$ & - & - \\
\hline bDES (ng/ml) & - & - & $0.17(0.12-0.23)$ & $0.30(0.21-0.37) \dagger$ \\
\hline
\end{tabular}

Data are shown as median (IQR) or mean \pm SD.

Note that healthy volunteers recruited for urine and sputum analysis (HV2a) were different from those for blood analysis (HV2b).

*A total of 47 patients with 'during an exacerbation' COPD were the same as those who had urine, sputum and blood collected.

$t p<0.001$, versus healthy volunteers, Mann-Whitney test.

bDES, blood desmosine; $\mathrm{FEV}_{1}$, forced expiratory volume in $1 \mathrm{~s}$; uDES, urinary desmosine. 
A


Figure 1 Urinary and blood desmosine (uDES and bDES) levels in patients with stable asthma, stable chronic obstructive pulmonary disease (COPD), 'during an exacerbation' COPD and healthy volunteers. uDES $(A)$ and bDES levels $(B)$ were analysed in a total of 390 participants from six subgroups including healthy non-smokers, healthy smokers, non-smokers with stable asthma, smokers with stable asthma, patients with stable COPD and patients with 'during an exacerbation' COPD. ${ }^{* *} p<0.001$, patients with 'during an exacerbation' COPD versus healthy volunteers. $\# p<0.001$, patients with stable COPD versus healthy non-smokers, healthy smokers, nonsmokers with asthma or smokers with asthma. The boundary of the box indicates the 25th and 75th percentile and the line within the box marks the median. Whiskers indicate the 90th and 10th percentiles. Note that healthy volunteers in group 2 recruited for uDES analysis were different from those for bDES analysis (table 1). (C) The relationship between UDES and bDES in healthy non-smokers, smokers, patients with stable COPD (from group 1) and patients with 'during an exacerbation' COPD (from group 2). The correlation coefficient and $p$ values expressed as $r, p$ for Spearman correlation were $0.53,<0.001 ; 0.18,0.33 ; 0.50,<0.001$; and $0.38,0.01$ for healthy non-smokers, healthy smokers, patients with stable COPD, and patients with 'during an exacerbation' COPD, respectively. correlation) and 'during an exacerbation' COPD ( $\mathrm{r}=0.38$, $\mathrm{p}=0.01$ ) (figure 1C). These results suggest that elevated bDES levels are specific to COPD and occur when patients are clinically stable or at the time of an exacerbation.

Increased bDES levels, however, were not observed in all patients with COPD (figure 1). Twenty-two of 52 (42\%) patients with stable COPD showed an elevated bDES concentration based on a nominal cut-off of $0.30 \mathrm{ng} / \mathrm{ml}$ (defined by the upper limit of a $95 \%$ prediction interval for desmosine values in all healthy non-smokers) on at least one visit. Among these patients, 17 of $48(35 \%)$ appeared to express elevated levels of bDES on both visits despite the intra-subject variability being significantly increased (see online supplementary figure E1). A similar observation was found in the 'during an exacerbation' COPD group in which 46 of 102 (45\%) had values above the cut-off.

\section{The association among uDES levels, proteinuria and lung inflammation in patients with 'during an exacerbation' COPD}

We postulated that the cause of higher urinary excretion of degraded elastin products in patients with 'during an exacerbation' COPD is due to compromised renal capillary endothelial permeability. Among factors occurring during COPD exacerbations, acute inflammation is known to affect renal capillary endothelial permeability leading to proteinuria or albuminuria. ${ }^{19}{ }^{20}$ To investigate this possibility, we measured MMP-9 activity, a parameter known to be associated with acute airway inflammation, ${ }^{21}$ in the sputum samples taken from the same patients during the same visit. As expected, a much higher (on average 10-fold) sputum MMP-9 activity was observed in patients with 'during an exacerbation' COPD compared with healthy volunteers in group 2 $(p<0.001$, figure $2 \mathrm{~A})$ while patients with stable COPD only showed a moderate $36 \%$ increase compared with healthy volunteers in group $1(p=0.05$, figure $2 A)$. The increased sputum MMP-9 activity correlated with the uDES concentration (figure $2 \mathrm{~B}$ ). The results support the hypothesis that many patients with 'during an exacerbation' COPD had an acute inflammatory response. In line with this hypothesis, urinary protein concentration in these patients was also found to be significantly higher $(p<0.001$, compared with healthy volunteers and patients with stable COPD, figure $2 \mathrm{C}$ ), whereas there were no differences among the stable COPD and other subgroups in group $1(p=0.7-1.0)$. In addition, we found that urinary protein concentration in all groups was highly correlated with non-normalised uDES level $(\mathrm{r}=0.79, \mathrm{p}<0.0001$, Spearman correlation, figure 2C). When the normalisation of $\mathrm{UDES}$ is based on urinary protein concentration (instead of creatinine as normally performed), no difference between healthy volunteers and patients with 'during an exacerbation' COPD was observed ( $p=0.97$, figure $2 \mathrm{D}$ ).

\section{Intra-subject variability of uDES and bDES levels}

Intra-subject variability in uDES and bDES levels between the two visits over a period of 6-8 weeks was assessed using CV and Bland Altman plot for each participant. The intra-subject variability of bDES was relatively smaller (average $\mathrm{CV}=6-12 \%$ ) compared with uDES (41-50\%) in healthy volunteers and patients with COPD (see online supplementary figure E1). The Bland-Altman plots suggest that the bDES levels were stable between visits and that the limits of agreement may be different (in absolute terms) between the groups.

\section{Association of bDES with clinical variables}

The potential correlations of bDES with clinical variables (age, gender, smoking pack years, blood cotinine levels, $\mathrm{FEV}_{1}$ 
Figure 2 The elevated urinary desmosine $(\mathrm{uDES}) /$ creatinine level in patients with 'during an exacerbation' chronic obstructive pulmonary disease (COPD) is associated with elevated urinary protein and increased sputum matrix metalloproteinase-9 (MMP-9) activity. (A) Sputum MMP-9 activity (normalised to total protein concentration) was measured in healthy volunteers and patients with COPD in groups 1 and $2 ;{ }^{*} p=0.05,+p<0.001$, Mann-Whitney $U$ test. Note that healthy volunteers in group 1 include healthy smokers and non-smokers (table 1). The boundary of the box indicates the 25th and 75th percentile and the line within the box marks the median. Whiskers indicate the 90th and 10th percentiles. (B) The relationship between sputum MMP-9 and UDES levels in healthy volunteers (group 2, HV2a), patients with stable COPD and patients with 'during an exacerbation' COPD. A significant correlation was found between these two measurements when all participants were included $(r=0.57, p<0.001$, Spearman correlation). (C) The un-normalised uDES level was highly correlated with urinary protein concentration $(\mathrm{p}<0.001, \mathrm{r}=0.78,0.63,0.49$ for healthy volunteers (group 2, HV2a), patients with stable COPD and patients with 'during an exacerbation' COPD, respectively; Spearman correlation). Urinary protein concentration was significantly elevated in patients with 'during an exacerbation' COPD in group 2 ( $p<0.001$ vs healthy volunteers, Mann-Whitney $U$ test). (D) No difference was observed between healthy volunteers and patients with 'during an exacerbation' COPD in group 2 when uDES levels were normalised to urinary protein levels; $p=0.97$, two sample $t$ test. The top boundary of the bar indicates the mean and the error bar indicates the SD.

(\% predicted), $\mathrm{FEV}_{1} / \mathrm{FVC}$ and transfer factor corrected for haemoglobin and carboxy haemoglobin (TLCO\%COHb) were examined. Age and TLCO\%COHb were the only two variables significantly associated with bDES levels. bDES levels were positively correlated with age in healthy volunteers $(r=0.42$, $\mathrm{p}=0.02$, Spearman correlation), in patients with stable COPD $(\mathrm{r}=0.54, \mathrm{p}<0.001)$ and in patients with exacerbated COPD $(\mathrm{r}=0.48, \mathrm{p}<0.001)$ (figure 3$)$. The age-related association was also found in smokers with asthma but did not reach statistical significance in non-smokers with asthma ( $r=0.34$ and 0.23 , $\mathrm{p}=0.01$ and 0.08 , respectively).

$\mathrm{TLCO} \% \mathrm{COHb}$ was also found to be negatively associated with bDES levels in patients with stable COPD $(r=-0.31$, $\mathrm{p}=0.02$, partial Spearman correlation controlled for age) (figure 4). In the non-smokers and smokers with asthma, the association was not significant $(\mathrm{p}=0.21$ and 0.55 , respectively, age controlled).

We did not observe a significant correlation between $\mathrm{FEV}_{1}$ (\% predicted) and bDES in patients with stable or 'during an exacerbation' COPD (see online supplementary figure E2). The data suggest that bDES levels may represent disease activity at the time rather than disease severity as assessed by baseline $\mathrm{FEV}_{1}$.

\section{DISCUSSION}

In the current study, we evaluated the clinical validity of bDES and $\mathrm{UDES}$ as biomarkers for COPD in the largest study of its kind to date, using validated isotope dilution LC-MS/MS methods. With the advantages in analytical validity of assays and study size, we demonstrated that the elevation of bDES and uDES levels was specific to COPD (but not asthma or smoking status); approximately $40 \%$ of patients had elevated bDES levels in stable COPD and during exacerbation groups; the association between bDES levels and decreased diffusing capacity of the

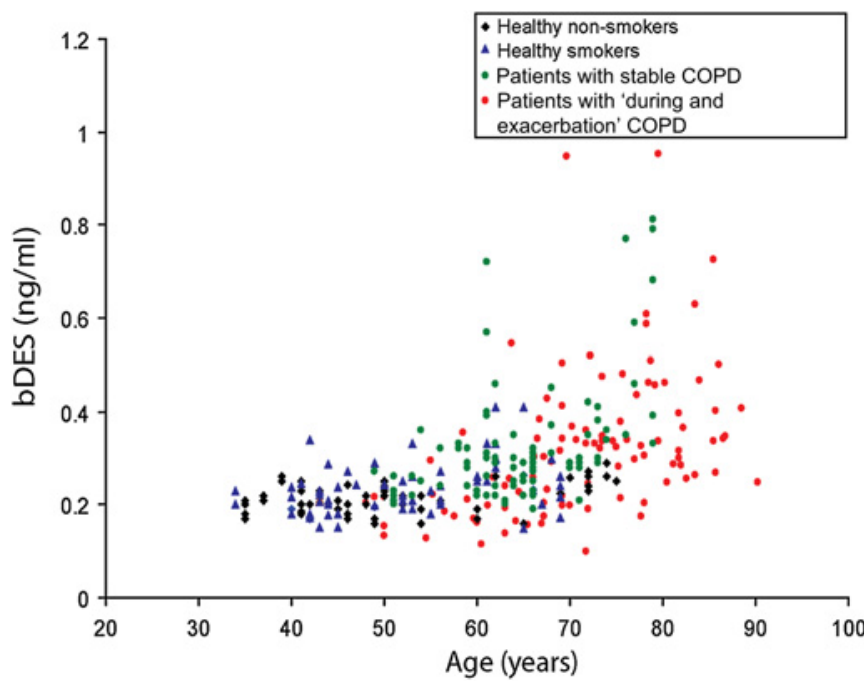

Figure 3 The relationship of age and blood desmosine (bDES) levels in healthy non-smokers, healthy smokers, patients with stable chronic obstructive pulmonary disease (COPD) and patients with 'during an exacerbation' COPD. The correlation coefficients for healthy volunteers, patients with stable COPD and patients with 'during an exacerbation' COPD were $0.42,0.54$ and 0.48 , respectively (Spearman correlation). The $p$ values were all $<0.001$. Note that there was a steeper gradient and higher 'patient-to-patient' variation in patients with stable COPD and 'during an exacerbation' COPD compared with healthy volunteers. 


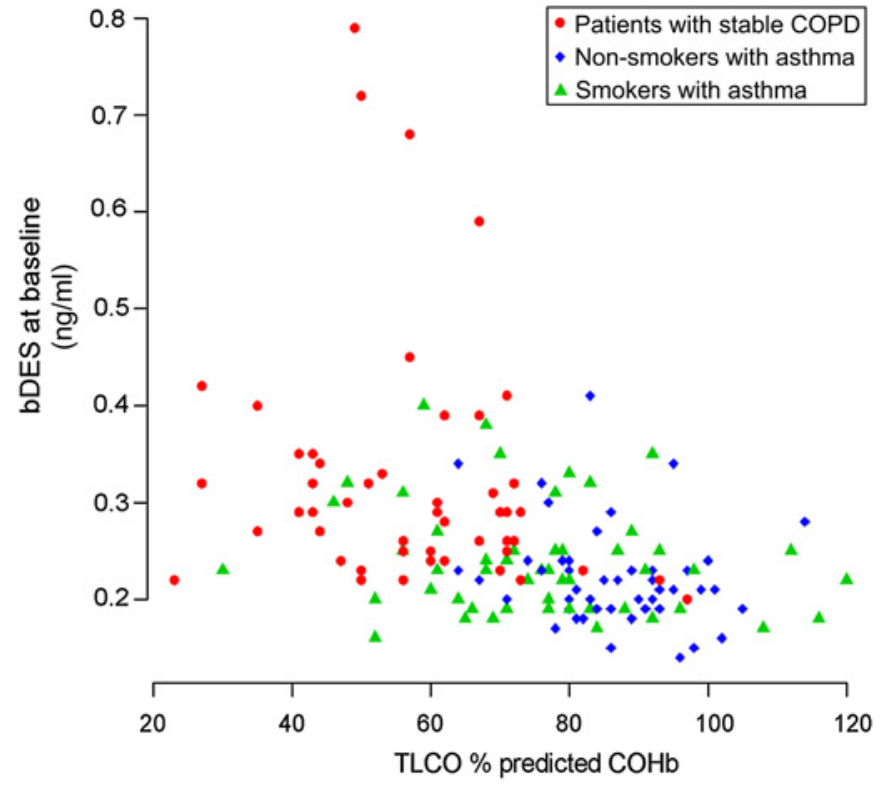

Figure 4 The relationship between blood desmosine (bDES) and the diffusing capacity for carbon monoxide (transfer factor of the lung for carbon monoxide corrected for haemoglobin and carboxy haemoglobin (TLCO \% predicted $\mathrm{COHb}$ )) was negatively associated with bDES levels in patients with stable chronic obstructive pulmonary disease (COPD) $(r=-0.31, p=0.02$, partial correlation (Spearman) controlled for age). In the non-smokers and smokers with asthma, the negative association was not significant $(\mathrm{p}=0.21$ and 0.55$)$.

lungs in the stable COPD group points to a potential link between this blood biomarker and lung pathophysiology; in contrast to bDES, uDES levels were associated with patients' exacerbation status or with proteinuria which we speculate might be caused by acute inflammation during the course of an exacerbation.

The interest in using DES/IDS as a surrogate endpoint in clinical trials for COPD has recently been revived after the previously unsuccessful attempts to use uDES in the determination of therapeutic response (or efficacy) following drug treatments in patients with $\mathrm{COPD}^{4} 22$ and $\alpha 1$-anti-trypsin deficiency. ${ }^{23} 24$ The growing interest is mainly due to the technical feasibility of measuring plasma and sputum DES/IDS levels, which requires assays of high sensitivity and specificity. ${ }^{25-27}$ In particular, $\mathrm{Ma}$ and colleagues' recent study showed that the long-acting anti-muscarinic bronchodilator tiotropium bromide reduced the DES/IDS levels in sputum and blood but not (total) uDES levels in patients with COPD over a 2 -month period. ${ }^{25}$ Despite there being a potential bias in the LC-MS/MS method used in that study (as no internal standard was used), the results support the view that bDES (and perhaps sputum DES) is a better surrogate endpoint biomarker than uDES. In those earlier studies ${ }^{6}{ }^{22-24}$ where uDES levels were measured to test drug efficacy in patients with clinically stable COPD, it is possible that the negative results observed were simply a combined result of the 'wrong' matrix (urine) and methods used. Without compromised renal capillary endothelial permeability, changes of elastin degradation over time may not be detectable in urine.

Our study suggests that approximately $40 \%$ of patients with COPD regardless of exacerbation status have elevated bDES levels based on a cut-off of $0.30 \mathrm{ng} / \mathrm{ml}$. We speculate that these patients may have increased elastin degradation based on the positive association with diffusing capacity for carbon monoxide. DES-containing and IDS-containing peptides have been shown to be rapidly accumulated in kidney and slowly released to urine in animal models. ${ }^{28}$ As we did not find signs of compromised renal function, for example, increased urinary protein/creatinine ratio in patients with stable COPD, we do not believe it was the cause of increased bDES levels. Targeting such a subgroup may have clinical significance in disease prognoses or the selection of treatment strategy, especially for the compounds targeting proteases and inflammation. The site of origin of abnormal elastin degradation in those $40 \%$ of patients with COPD remains to be investigated. Patients with COPD also have comorbidities such as cardiovascular disease, which may potentially affect bDES levels as mature elastin is highly expressed in blood vessels. ${ }^{29}$ To be able to use bDES as a surrogate endpoint and prognostic biomarker, further large longitudinal studies are needed to demonstrate the relationship between its levels and long-term clinical outcomes, particularly in the decline of lung function and development of emphysema.

It is unexpected and interesting that bDES levels in patients with COPD during an exacerbation did not differ from those of patients with stable COPD despite the fact that many of these patients had increased airway inflammation as suggested by the elevated sputum MMP-9 activity. One potential explanation is that an increased filtration rate to the urine during an exacerbation helps the clearance of degraded elastin products in the blood. Consequently, the bDES levels observed in patients with COPD during an exacerbation could be underestimated. The sum of blood and urinary desmosine levels (multiplied by the volume) may be a useful index to estimate total elastin breakdown.

Urinary DES and bDES concentrations in patients with chronic asthma, including a subgroup who were smokers, were not elevated, which is in keeping with the finding that most patients with asthma do not develop emphysema. Whether exacerbations of asthma, particularly in those who smoke, increase $\mathrm{uDES}$ and bDES concentrations was not investigated in this study.

We recognise that there were limitations in this study, such as its cross-sectional nature, limited visit numbers and confounding factors, for example, corticosteroid and/or antibiotic treatment during admission in the 'during an exacerbation' COPD group. Further investigation in a longitudinal cohort is required with baseline measurements and then during and after an exacerbation.

In summary, this study has demonstrated that the elevation of $\mathrm{UDES}$ or bDES levels was only significant in patients with COPD, but not in those with asthma or healthy smokers. The elevation of UDES level, however, was only observed during an exacerbation of COPD but not in stable COPD. We found that blood desmosine levels are elevated in a subgroup of approximately $40 \%$ of patients with stable COPD. We speculate that a raised blood desmosine level may identify patients with increased elastin degradation suitable for targeted therapy. Future prospective studies are required to investigate this hypothesis.

Acknowledgements The authors would like to thank Ms Lorna Gillespie for technical support, Joyce Thompson, Jane Lafferty, Maureen Brannigan, Iona Donnelly and Lisa Jolly for sample collection and storage. The research team also acknowledges the financial support of NHS Research Scotland (NRS), through Scottish Primary Care Research Network for assistance with recruitment.

Funding This work was funded by awards DU106 and GU90 from the Translational Medicine Research Collaboration - a consortium made up of the Universities of 
Glasgow, Edinburgh, Aberdeen and Dundee and the four associated NHS Health Boards (Greater Glasgow \& Clyde, Lothian, Grampian and Tayside), Scottish Enterprise and Pfizer (formerly Wyeth) and supported financially by NHS Research Scotland, through the Scottish Primary Care Research Network.

Competing interests $\mathrm{JH}, \mathrm{GF}, \mathrm{JB}$ and DM were employed by Pfizer Inc. during the study. This does not alter their adherence to all the Thorax policies.

Patient consent Obtained.

Ethics approval Ethics approval was provided by ethics committees and Institutional Review Board (Glasgow and Dundee).

Contributors Conception and design: JH, RC, GF, SM, JB, CP, DM, NT; analysis and interpretation: $\mathrm{JH}, \mathrm{RC}, \mathrm{AB}, \mathrm{CG}, \mathrm{OA}, \mathrm{PR}, \mathrm{CW}, \mathrm{MM}, \mathrm{CM}, \mathrm{NS}, \mathrm{DM}, \mathrm{NT}$; drafting the manuscript for important intellectual content: JH, RC, NT.

Provenance and peer review Not commissioned; externally peer reviewed.

\section{REFERENCES}

1. Agusti A, Calverley PM, Celli B, et al. Characterisation of COPD heterogeneity in the ECLIPSE cohort. Respir Res 2010:11:122.

2. Sharafkhaneh $\mathbf{A}$, Hanania NA, Kim V. Pathogenesis of emphysema: from the bench to the bedside. Proc Am Thorac Soc 2008;5:475-7.

3. Miao M, Bruce AE, Bhanji T, et al. Differential expression of two tropoelastin genes in zebrafish. Matrix Biol 2007;26:115-24.

4. Luisetti M, Ma S, ladarola P, et al. Desmosine as a biomarker of elastin degradation in COPD: current status and future directions. Eur Respir $J$ 2008; 32:1146-57.

5. Cocci F, Miniati M, Monti S, et al. Urinary desmosine excretion is inversely correlated with the extent of emphysema in patients with chronic obstructive pulmonary disease. Int J Biochem Cell Biol 2002;34:594-604.

6. Luisetti M, Sturani C, Sella D, et al. MR889, a neutrophil elastase inhibitor, in patients with chronic obstructive pulmonary disease: a double-blind, randomized, placebo-controlled clinical trial. Eur Respir J 1996:9:1482-6.

7. McClintock DE, Starcher B, Eisner MD, et al. Higher urine desmosine levels are associated with mortality in patients with acute lung injury. Am J Physiol Lung Cell Mol Physiol 2006:291:L566-71.

8. Starcher B, Green M, Scott M. Measurement of urinary desmosine as an indicator of acute pulmonary disease. Respiration 1995;62:252-7.

9. Annovazzi L, Viglio S, Perani E, et al. Capillary electrophoresis with laser-induced fluorescence detection as a novel sensitive approach for the analysis of desmosines in real samples. Electrophoresis 2004;25:683-91.

10. Viglio S, ladarola P, Lupi A, et al. MEKC of desmosine and isodesmosine in urine of chronic destructive lung disease patients. Eur Respir J 2000;15:1039-45.

11. Fiorenza D. Viglio $S$, Lupi $A$, et al. Urinary desmosine excretion in acute exacerbations of COPD: a preliminary report. Respir Med 2002:96:110-14.
12. Stolk J, Veldhuisen B, Annovazzi L, et al. Short-term variability of biomarkers of proteinase activity in patients with emphysema associated with type Z alpha-1-antitrypsin deficiency. Respir Res 2005;6:47.

13. Viglio S, Zanaboni G, Luisetti M, et al. Micellar electrokinetic chromatography for the determination of urinary desmosine and isodesmosine in patients affected by chronic obstructive pulmonary disease. J Chromato B Biomed Sci Appl 1998;714:87-98.

14. Stone PJ, Konstan MW, Berger M, et al. Elastin and collagen degradation products in urine of patients with cystic fibrosis. Am J Respir Crit Care Med 1995:152:157-62.

15. Stone PJ, Bryanrhadfi J, Lucey EC, et al. Measurement of urinary desmosine by isotope dilution and high performance liquid chromatography. Correlation between elastase-induced air-space enlargement in the hamster and elevation of urinary desmosine. Am Rev Respir Dis 1991;144:284-90.

16. Cumiskey WR, Pagani ED, Bode DC. Enrichment and analysis of desmosine and isodesmosine in biological fluids. J Chromato B Biomed Sci App/ 1995;668:199-207.

17. Stone PJ, Gottlieb DJ, O'Connor GT, et al. Elastin and collagen degradation products in urine of smokers with and without chronic obstructive pulmonary disease. Am J Respir Crit Care Med 1995; 151:952-9.

18. Albarbarawi $\mathbf{0}$, Barton A, Lin Z, et al. Measurement of urinary total desmosine and isodesmosine using isotope-dilution liquid chromatography-tandem mass spectrometry. Anal Chem 2010;82:3745-50.

19. Cogo A, Ciaccia A, Legorini $\mathrm{C}$, et al. Proteinuria in COPD patients with and withou respiratory failure. Chest 2003:123:652-3; author reply 3 .

20. Abid 0, Sun Q, Sugimoto K, et al. Predictive value of microalbuminuria in medical ICU patients: results of a pilot study. Chest 2001;120:1984-8.

21. Warner RL, Bhagavathula N, Nerusu KC, et al. Matrix metalloproteinases in acute inflammation: induction of MMP-3 and MMP-9 in fibroblasts and epithelial cells following exposure to pro-inflammatory mediators in vitro. Exp Mol Pathol 2004; 76:189-95

22. Cohen $\mathbf{A B}$, Girard W, McLarty J, et al. A controlled trial of colchicine to reduce the elastase load in the lungs of ex-cigarette smokers with chronic obstructive pulmonary disease. Am Rev Respir Dis 1991;143:1038-43.

23. Gottlieb DJ, Luisetti M, Stone PJ, et al. Short-term supplementation therapy does not affect elastin degradation in severe alpha(1)-antitrypsin deficiency. The American-Italian AATD Study Group. Am J Respir Crit Care Med 2000;162:2069-72.

24. Stoller JK, Rouhani F, Brantly M et al. Biochemical efficacy and safety of a new pooled human plasma alpha(1)-antitrypsin, Respitin. Chest 2002;122:66-74.

25. Ma S, Lin YY, Turino GM. Measurements of desmosine and isodesmosine by mass spectrometry in COPD. Chest 2007:131:1363-71.

26. Ma S, Lin YY, Tartell L, et al. The effect of tiotropium therapy on markers of elastin degradation in COPD. Respir Res 2009;10:12.

27. Ma S, Lieberman S, Turino GM, et al. The detection and quantitation of free desmosine and isodesmosine in human urine and their peptide-bound forms in sputum. Proc Natl Acad Sci U S A 2003;100:12941-3.

28. Starcher B, Peterson B. The kinetics of elastolysis: elastin catabolism during experimentally induced fibrosis. Exp Lung Res 1999;25:407-24.

29. Armentano RL, Levenson J, Barra JG, et al. Assessment of elastin and collagen contribution to aortic elasticity in conscious dogs. Am J Physiol 1991;260:H1870-7.

\section{Thorax alerts}

Sign up for our electronic table of contents alerts and you will never miss new issues of Thorax when published online. Stay ahead and up to date by visiting thorax.bmj.com. 